\title{
To evaluate efficacy and complications of suction drains in hemiarthroplasty cases
}

\author{
Vijay $\mathrm{C}^{1, *}$, Ravikiran $\mathrm{HG}^{2}$, Sumit Raj $^{3}$ \\ ${ }^{1}$ Associate Professor, ${ }^{2}$ Assistant Professor, ${ }^{3}$ Post Graduate, Dept. of Orthopaedics, JSS Hospital, Mysore, Karnataka, India
}

*Corresponding Author: Vijay C

Email: jssmc09@gmail.com

\begin{abstract}
Introduction: Intracapsular fracture of neck femur usually present with severe swelling, difficulty in walking reduced length of affected limb. The most common Risk factors are old age, osteoporosis, taking excessive medications, alcohol, smoking and cancer. Diagnosis can be done by using X-rays, CT scan and MRI.

Materials and Methods: It is a prospective study consists of all the patients admitted to JSS hospital with intracapsular fracture neck femur treated with unipolar or bipolar endoprosthesis.

Results: The selected patients were randomized and divided into two groups, Group one (I) and Group two (II). Patients were suction tube is used are categorized into Group I and in those were suction tube is not used are categorized into Group II. These two groups were studied and observed for the following, fall in haemoglobin and PCV at 48 hours post-operatively. Complications are Persistent discharge, serous discharge from the wound for more than 10 days.

Discussion: When the doctors are in doubt, then they need to insert a drain was countered by Halsted (1898). Now days in orthopedic cases, closed suction drains are being commonly used to decrease the complications. Blood accumulation in and around the tissue site will raise the tissue tension and thereby reduces tissue perfusion in and around the surgical wound site.

Conclusion: We consider that there is ample evidence that closed suction drains is of no benefit in primary uncomplicated hemiarthroplasty. The advantage of the drains in orthopaedic surgeries should be debated as non use of drains drastically reduces anemia and requirement of blood transfusion after surgery. In a developing and under-developing countries, the cost increases because of the blood transfusion needs and costs of drain device which will outweigh any advantages of putting a drain.
\end{abstract}

Keywords: Persistent discharge, Dressing reinforcements, Superficial surgical site infections, Blood transfusion.

\section{Introduction}

Intracapsular fracture of neck femur usually present with severe swelling, difficulty in walking reduced length of affected limb. The most common Risk factors are old age, osteoporosis, osteopenia, taking excessive medications, alcohol, smoking and cancer. Diagnosis can be done by using X-rays, CT scan and MRI and bone scan. Opiods are the best drug of choice to reduce the pain by nerve block. The main surgery of choice is THR, hemiarthroplasty or internal fixation with cannulated cancellous screws. About $15 \%$ of women will land up fracture neck of the femur. Women are more often affected than men. Hip fractures become more common with age. The chance of death in the year after a fracture is about $20-25 \%$ in older people.

Total hip replacement is done very commonly done surgical procedure to relieve pain, correct deformity and to gain good function and good range of movement of the joint. There is extensive soft tissue damage and bleeding during the surgery because bony osteotomy, is done and hemorrhage is more postoperatively which is a major concern and which would lead to anemia and necessitate blood transfusion intraoperative and postoperatively. Blood transfusion is relatively difficult procedure if the transfusion medicine department falls short of blood products and also there is a risk of infections secondary to transfusion, our cultural aversion is also present for the blood transfusion, recently the use of blood transfusion in orthopedics surgeries are drastically reduced. ${ }^{1}$
Now a day's post-operative infections are reduced due to use of suction drainage during and following total hip replacement. But surgeons have repeatedly challenged the regular use of postoperative closed suction drainage. Putting a drain will effectively decrease the hematoma formation which will reduce postoperative pain, swelling, and the chances of infection. Meantime the reduction of hematoma formation will increase the postoperative hemorrhage by reducing the tamponade effect at the surgical wound site, which will raise the risk and need of blood transfusion. The drainage clamping may be good choice to reduce the blood loss and also the related complication of hematoma. Most of the time blood loss occurs during the first few hours of the postoperative phase (about 37\% in two hours and 55\% in four hours). The tamponade effect at the wound closure site about 4 hours of drainage clamping will reduce the blood loss in total hip replacement. ${ }^{2}$

Earlier studies have focused primarily on total knee arthroplasty rather than total hip replacement and hemiarthroplasty. Methodologies will vary with different types of continuous and intermittent clamping times during total knee replacements. If the clamping period increases there is increased risk of complications like delayed wound healing, hematoma formation, necrosis of the wound edges, and infection rates. Previous studies have claimed that the four hour drainage clamping method would achieve good result. A study has also confirmed that the four-hour drainage 
clamping method will reduce blood loss effectively during total knee arthroplasty. ${ }^{3}$

As far as we know, there have been only two studies that have previously evaluated drainage clamping in total hip arthroplasty. Brueggemann et al. also reported that the two suction drains clamped intermittently for 55 minutes every hour for about first six hours of postoperative phase would significantly reduces the blood loss. Recently Cao et al. also proved that a six-hour drainage clamping technique in total hiparthroplasty will reduce blood loss and reduction of postoperative drainage where the need for blood transfusion is decreased. Thinking uncertainty of this technique, the objective of this research is to find out the safety, efficacy of a four-hour drainage clamping method in patients undergoing hemiarthroplasty. ${ }^{3}$

\section{Materials and Methods}

It is a prospective study consists of all the patients admitted to JSS hospital with extracapsular fracture neck femur treated with unipolar or bipolar end prosthesis.

\section{Inclusion Criteria}

1. Male and female patients aged more than 50 years

2. Displaced intracapsular fracture neck of femur

3. Neglected cases of intracapsular fracture neck of femur

4. Nonunion of intracapsular fracture neck of femur

\section{Exclusion Criteria}

1. Intertrochanteric fractures

2. Fracture of the neck of the femur in young patients aged less than 50 years

3. Any other patients associated with any other ipsilateral or contra lateral fracture of upper and lower extremities.

4. Patient with neurological disorders.

5. Patient unfit for surgery.

6. Patient not willing for surgery.

7. Patients with uncontrolled diabetes mellitus (Hba1c $>7.0$ )

8. Patients on previous anticoagulation for underlying comorbidities.

All the consecutive patients operated with hemiarthroplasty were taken for the data which were operated between September 2015 to May 2017.Once the endoprosthesis was fit in the femoral canal and reduced after a good wash, hip capsule was closed with absorbable sutures and later short external rotators was reattached to the greater trochanter with ranawats type of closure after drilling the greater trochanter later a romovac suction drain tube drain of number 14 was fixed in the safe sites and wound was sutured in layers mainly the gluteus maximus, subucataneous layer and later skin sutured with either skin staples or with nonabsorbable sutures these were in the group of suction drain in situ after the hemiathroplasty where as in other group wound was closed in the similar fashion without romovac suction drain tube drain. Later both were the group of patients were followed up until the drain was removed and further and soakage was noted check dressing done meticulously and drain was removed after 48 hours or once drain collection was less than $50 \mathrm{ml}$ mean time in the drain group drain tip was sent for culture and sensitivity. Haemoglobin level was checked in both group of patients after 48 hours, if the hemoglobin level is decreased the same was noted and PRBC blood transfusion was started if the hemoglobin is less the 7 gram percent. Dressing reinforcement, patients requiring extra dressings and cost required as opposed to the routine dressing days due to soakage, superficial surgical site infections, Hematoma formation were noted. The mean fall in hemoglobin and PCV were calculated. The number of PRBC transfusions required in both the groups was observed.

\section{Results}

The selected patients were randomized and divided into two groups, Group one (I) and Group two (II). Patients were suction tube is used are categorized into Group I and in those were suction tube is not used are categorized into Group II. These two groups were studied and observed for the following: Fall in Haemoglobin and PCV at 48 hours post-operatively. Complications are Persistent discharge and wound discharge for more than 10 days.

Dressing reinforcement, patients requiring extra dressings as opposed to the routine dressing days due to soakage, superficial surgical site infections, Hematoma formation. The mean fall in hemoglobin and PCV were calculated. The number of PRBC transfusions required in both the groups was observed.

The mean fall in Haemoglobin was noted to be 3 gm\% in Group I whereas it was 2 gm\% in Group II and mean fall in PCV was 10\% in Group I and 7\% in Group II. Though difference in the two groups was noted but it was statistically insignificant. In Group I persistent discharge was seen in 4 patients whereas in Group II it was seen in 3 patients, the difference being statistically insignificant. In Group I dressing reinforcement was required in 6 patients and in Group II 9 patients needed dressing reinforcement which was statistically insignificant. Infection of surgical site was seen in 4 patients in Group I and in Group II only 2. One patient in Group II haematoma was seen whereas none of the patients in group I developed haematoma formation. Group I patients that is with the drain group 8 patients required PRBC transfusion whereas only 3 patients required PRBC transfusion in Group II postoperatively. This was found to be statistically significant. 


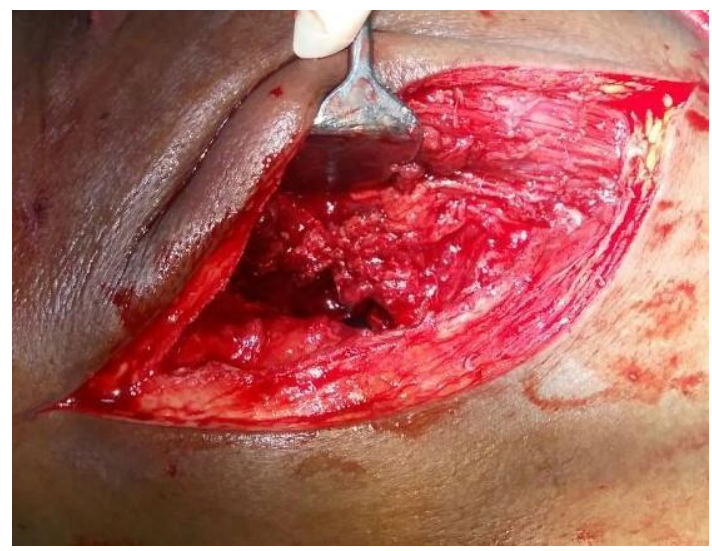

Fig. 1: Exposure of the hip joint after cutting the capsule

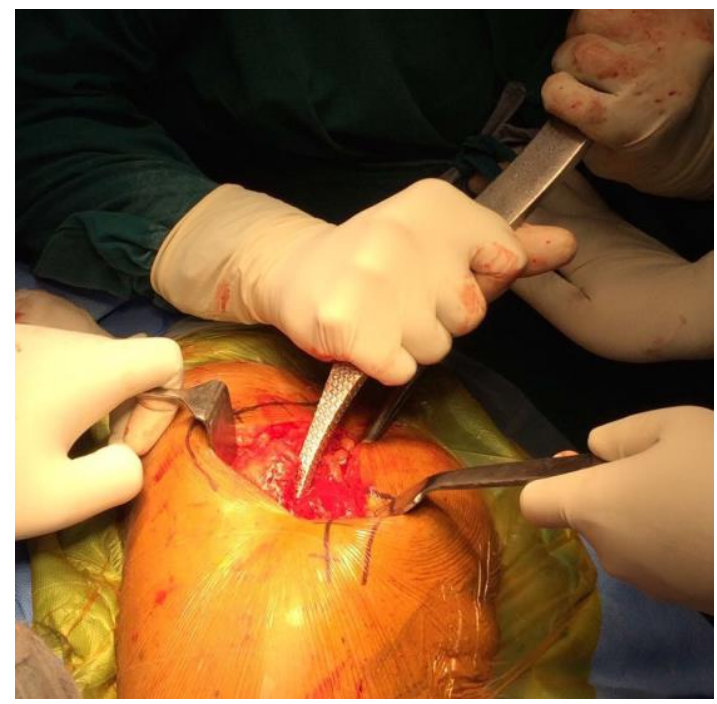

Fig. 2: Broaching of the femoral canal after the femoral head extraction

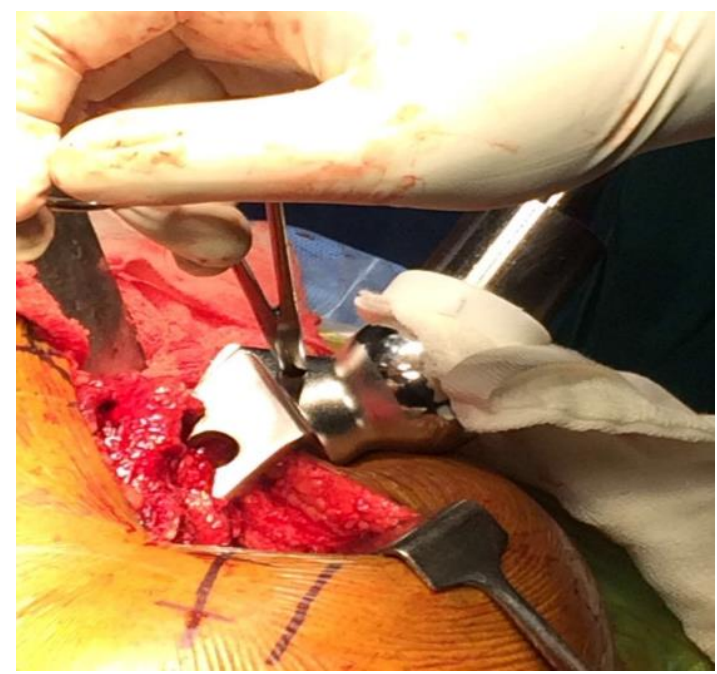

Fig. 3: AMP unipolar prosthesis inserted into the femoral canal

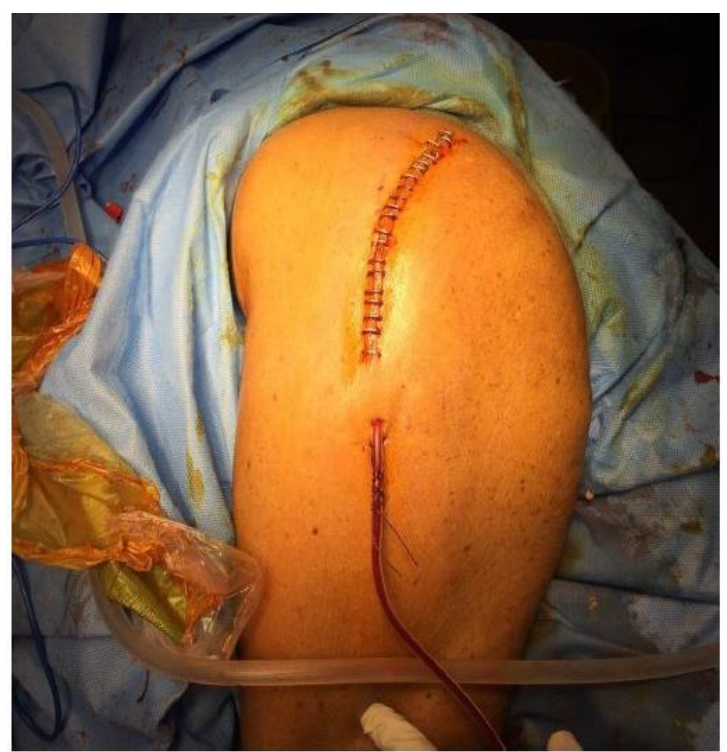

Fig. 4: Wound with closed suction drain

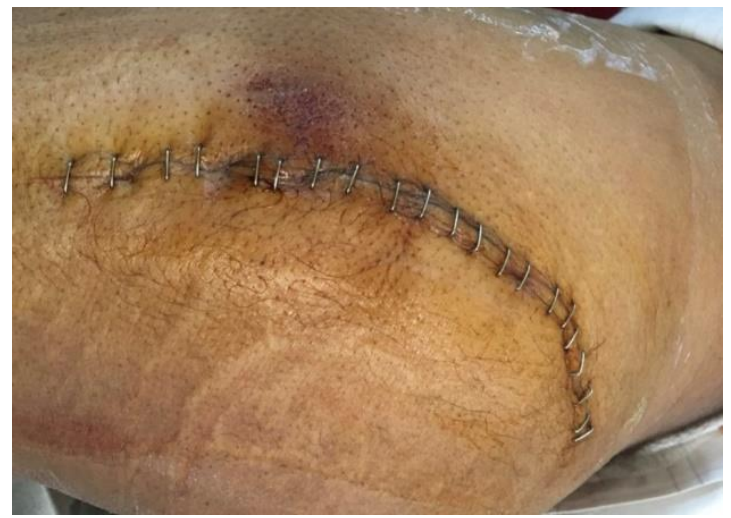

Fig. 5: Wound without closed suction drain

\section{Discussion}

When the doctors are in doubt, then they need to insert a drain was countered by Halsted (1898). Now days in orthopedic cases, closed suction drains are being commonly used to decrease the complications. Blood accumulation in and around the tissue site will raise the tissue tension and thereby reduces tissue perfusion in and around the surgical wound site.

This will effectively increase the chance of wound infection and decrease the chances of healing. The routine use of drains is still followed despite of proving drawbacks of its use. The many studies done in the worldwide have noted the disparity between literature and also the practice of using drain commonly among orthopaedic surgeons. Great number of studies have compared whether to use or not to use the closed suction drains in hemiarthroplasty and total joint arthroplasty. ${ }^{4}$

However, some studies done by Hadden and McFarlane have not been prospective and have evaluated only a few patients with a hip replacement. Moreover, the use of low molecular weight heparin for antithrombotic prophylaxis and its associated risk of 
excessive bleeding, has not always mentioned. Only few prospective, randomized trial study comprising more than 100 patients has been done in 1994 . Usually the drain will remove the postoperative hematoma and thus reducing the incidence of infections. But the recent prospective, randomized study done claims the use of a drain did not reduce the volume of the postoperative hematoma in total joint arthroplasty cases done by Widman et al in 2002. Because of suction tubes it can be cause of infection if the bacterial migration occurs along the tubes. ${ }^{5}$

In a prospective study done by Sørensen and Sørensen in 1991 detected bacterial growth in the drain tip of 56 patients out of which 5 among of them developed infection subsequently. This finding the development of superficial infections in 3 of our patients, in drained group as compared to 1 in nondrained group. In the present study, hemovac drains had been used. Persistent bleeding because of the negative pressure at the surgery site also tells us that there is marked decline in hematocrit in patients with closed suction drains which has been recently suggested by Widman et al in 2002. ${ }^{6}$

Ritter and coworkers did a randomized study in 1994 wherein 415 patients undergoing total hip or knee replacements for closed suction drain or no drainage, and they found no differences in the occurrence of infection between the two groups. Their surgical technique differed from ours since they did not routinely repair the posterior soft tissues, only minimal hypotension was induced, and all patients were given acetylsalicylic acid postoperatively and 10 patients were immobilized for a short period when persistent wound drainage was seen. In our study, no patient was kept in bed because of persistent drainage. We believe that some patients at high risk of thromboembolic disease may require anticoagulation with warfarin or low molecular weight heparin as suggested by $\mathrm{Di}$ Giovanni et al in 2000.7,8

Maximum care should be given to minimize the occurrence of haematom as infection. This will automatically reduces the chance of infection and other complications of the wound, and also the reduction in the need for reinforcement and the change of postoperative wound dressings as said by the proponents while opponents cite studies suggestive of drains actually increasing the risk of infection. There are many researches that do not support the routine use of prophylactic drainage to decrease the frequency of post operative wound complications in orthopaedic surgery. Our results show no statistical differences between the drained group and the undrained groups with respect to pain scores, wound discharge, change of dressing requirements and the swelling of the wound. So Prophylactic wound drainage will not provide clear advantages over a no drainage policy with respect to these parameters. Post operative use of blood transfusion rates was significantly more in the drain group and this was proved in the other studies. Decreasing the treatment cost is very important in developing and under-developed countries to increase the chance of availability of treatment to many patients.

\section{Conclusion}

We consider that there is ample evidence that closed suction drains is of no benefit in primary uncomplicated hemiarthroplasty. We consider that there is ample evidence that closed suction drains is of no benefit in primary uncomplicated hemiarthroplasty. The advantage of the drains in orthopaedic surgeries should be debated as non use of drains drastically reduces anemia and requirement of blood transfusion after surgery. In developing and under-developing countries, the cost increases because of the blood transfusion needs and costs of drain device which will outweigh any advantages of putting a drain.

\section{References}

1. Tai TW, Yang CY, Jou IM. Temporary drainage clamping after total knee arthroplasty: A meta-analysis of randomized controlled trials. $J$ Arthroplasty. 2010;25:1240-4.

2. Brueggemann P, Tucker J, Wilson P. Intermittent clamping of suction drains in total hip replacement reduces postoperative blood loss: A randomized, controlled trial. $J$ Arthroplasty. 1999;14:470-72.

3. Cao J-G, Wang L, Liu J. The use of clamped drainage to reduce blood loss in total hip arthroplasty. J Orthop Surg Res. 2015;10:130.

4. Loh A, Jones PA. Evisceration and other complications of abdominal drains. Postgraduate Medical Journal. 1991;67(789):687-8.

5. Ikpeme IA, Ngim NE, Ilori IU, Oku E, Udosen AM. Prophylactic wound drainage in orthopaedics: a comparative evaluation of closed suction drainage 85 versus no-drainage in a Nigerian teaching hospital. Surgical Science. 2013;4(05):277.

6. Tucci G, Amorese V, Romanini E. Closed suction drainage after orthopaedic surgery: evidence versus practice. Journal of Orthopaedics and Traumatology. 2006;7(1):2932.

7. Chandratreya A, Giannikas K, Livesley P. To drain or not drain: literature versus practice. Journal of the Royal College of Surgeons of Edinburgh. 1998;43(6):404-6.

8. Waugh TR, Stinchfield FE. Suction drainage of orthopaedic wounds. JBJS. 1961;43(7):939-1021.

How to cite this article: Vijay C, Ravikiran HG, Raj S. To evaluate efficacy and complications of suction drains in hemiarthroplasty cases. Indian J Orthop Surg. 2018;4(3):286-289. 